\title{
The Use of Neutrophil Gelatinase Associated Lipocalin (NGAL) As A Novel Marker in Early Diagnosis of Hepatorenal Syndrome in Advanced Cirrhotic Patients
}

Ahmed Samir Abo Halima, Nevine Ibrahim Musa, Amr Ahmed Okasha, Ahmed Samir Allam*

Department of Internal Medicine, Gastroenterology, Faculty of Medicine, Ain Shams University

*Corresponding author: Ahmed Samir Allam, Mobile: 01001701223,

Email: ahm82allam@gmail.com

\begin{abstract}
Background: Acute kidney injury (AKI) in cirrhosis, including hepatorenal syndrome (HRS), is a common and serious complication in cirrhotic patients, leading to significant morbidity and mortality.

Objective: To predict value of serum and urinary NGAL as an early predictor of hepatorenal syndrome in cirrhotic patients.

Patients and Methods: This study was a prospective clinical trial conducted at Kafr El Sheikh Liver Institution. The study included 60 participants with cirrhosis Child C on Child-Pugh score, each one was investigated for both Urinary and Plasma NGAL level.

Results: Group I had urinary NGAL ranged from $12-120$, with a mean value of $61.45 \pm 36.17$. While group II A, ranged from 211-493 with a mean value of 379 \pm 72.825 and in group II B, ranged from 306-732 with a mean value of $438.05 \pm 111.26$. There was statistical difference between studied groups, $\mathrm{p}$ value $<0.001$. It was found that urinary NGAL is not only good predictor for early renal affection, but also it can differentiate between HRS and non HRS AKI due to the marked difference readings of its level in groups with renal impairment.

Conclusion: Urinary NGAL shows noticeable differences between degree of elevation with different causes of AKI, so it somehow could be a trusted diagnostic tool to diagnose HRS.
\end{abstract}

Keywords: Acute kidney injury, HRS, NGAL.

\section{INTRODUCTION}

In individuals with liver cirrhosis, kidney impairment is a complicated and prevalent occurrence. Acute kidney damage (AKI) is a significant consequence of decompensated liver cirrhosis, with high morbidity and death rates. Despite some hopeful findings from new therapies ${ }^{(1)}$, AKI remains a major complication of decompensated liver cirrhosis with high morbidity and mortality rates. Prerenal azotemia (PRA), hepatorenal syndrome (HRS), and acute tubular necrosis (ATN) are among the most common causes of AKI in hospitalised patients with liver cirrhosis, with prevalence rates of about 68 percent, 25 percent, and 33 percent, respectively. According to studies, about $1 \%$ of cirrhotic individuals with azotemia develop progressive parenchymal renal disease as a result of hepatic viral infections, immunological, or metabolic problems (chronic glomerulonephritis, IgA nephropathy, diabetic nephropathy) ${ }^{(\mathbf{1}, 2)}$.

Members of the Acute Dialysis Quality Initiative (ADQI) and the International Ascites Club (IAC) collaborated in 2011 to establish a new set of diagnostic criteria for better evaluating kidney impairment in liver cirrhosis ${ }^{(3)}$. The term AKI refers to a rapid loss in renal function, as evidenced by a rise in serum creatinine levels of more than $50 \%$ from baseline in less than 48 hours, or an upward trend in serum creatinine levels of $\geq 26.4 \mathrm{~mol} / \mathrm{L}(0.3 \mathrm{mg} / \mathrm{dL})$ in less than 48 hours. Using the Modification of Diet in Renal Disease 6 (MDRD6) formula, chronic kidney disease (CKD) is defined as an estimated glomerular filtration rate (eGFR) of less than $60 \mathrm{ml} /$ minute for more than 3 months. According to the previous criteria for AKI and $\mathrm{CKD}$, acute chronic kidney disease appears as an overlap of AKI with pre-existing chronic renal disease (4).

The members of the ADQI group met in Vicenza (Italy) in May 2002 to develop a new set of diagnostic and classification criteria for AKI: the RIFLE classification (published in May 2004). This approach divides patients into three severity categories (Risk, Injury, and Failure) and two outcome categories (Loss of kidney function and end-stage renal disease) based on changes in blood creatinine, glomerular filtration rate, or urine output ${ }^{(5)}$.

The Acute Kidney Injury Network (AKIN) group established a new set of AKI criteria called the AKIN classification during a conference in Amsterdam in September 2005 (Published in March 2007). This enhanced the categorization method, which was based only on changes in serum creatinine (two measurements within 48 hours) and urine output and consisted of three levels of severity ${ }^{(5)}$.

The failure of both categorization methods to offer any information on the etiology of renal impairment in liver cirrhosis is a frequent flaw. Existing evidence does not support the AKIN classification's advantage over standard risk prediction criteria in patients with liver cirrhosis and renal failure (6). Fagunds et al. ${ }^{(7)}$ note that the combination of the AKIN classification and conventional criteria for renal impairment may provide a better risk assessment in patients with cirrhosis, compared to the AKIN criteria 
alone. Although its inaccuracy, particularly in the presence of liver cirrhosis, serum creatinine remains our current and most commonly used measure for kidney disease. Reduced hepatic production of creatinine and substantial loss of muscle mass in individuals with liver cirrhosis and CKD can lead to an overestimation of GFR when utilising creatinine-based formulae. After a renal insult occurs in the setting of AKI, a steady state must be achieved in order to correctly estimate GFR using serum creatinine ${ }^{(8)}$.

The purpose of our study was to predict value of serum and urinary NGAL as an early predictor of hepatorenal syndrome in cirrhotic patients.

\section{PATIENTS AND METHODS}

This study was a prospective clinical trial conducted at Kafr El Sheikh Liver Institution. The study included 60 participants with cirrhosis Child $\mathrm{C}$ on Child-Pugh score, each one was investigated for both urinary and plasma NGAL level. Plasma NGAL done using $5 \mathrm{ml}$ blood, while urinary NGAL done by using single urine sample for each patient. Serum NGALlevels assessed in the plasma of healthy adults normally is up to $300 \mathrm{ng} / \mathrm{mL}$. While urinary NGAL of healthy adults normally is up to 132 .

\section{Patients were divided into two main groups:}

Group (1): Control group (20 patients), cirrhotic, Child $\mathrm{C}$ on Child -Pugh score, without renal affection, normal serum creatinine, urea and urine analysis.

Group (2): 40 cirrhotic patients with renal impairment, who went under further subdivision into two subgroups: Group 2 A: 20 patients with known reason for renal impairment as contrast nephropathy, pigment nephropathy, dehydration, acute severe gastroenteritis, recent GI bleeding, drug induced, urinary tract infection, etc..., by talking detailed history and performing lab investigations as mentioned below. Group 2 B: 20 patients with renal impairment fulfilling diagnostic criteria of HRS.

Clear history regarding recent use of nephrotoxic drugs (non-steroidal anti-inflammatory drugs, aminoglycosides, iodinated contrast media, etc.), free both kidneys with ultrasound, cirrhotic with ascites, no response after 2 consecutive days of diuretic withdrawal and plasma volume expansion with albumin ( $1 \mathrm{~g} / \mathrm{kg}$ of body weight), absence of shock, no current or no macroscopic signs of structural kidney injury, no proteinuria, no microhematuria.

Exclusion Criteria: Patients known to have CKD. Liver transplant patients. Kidney transplant patients. The patients who refused to be entitled in the study.

\section{Ethical Considerations:}

All procedures performed in this study were in accordance with the ethical standards of Ain Shams University Research Committee and with the 1964 Helsinki declaration and its later amendments. Ethics Committee's reference number: 000017585. Faculty of Medicine, Ain Shams University, Cairo 11211, Egypt. Informed written consent was obtained from each participant before enrolment in the study.

\section{All patients were subjected to the following:}

Full history taking. Full clinical examination. Abdominal ultrasound. Laboratory investigations: Complete Blood Count. Serum Creatinine. Serum Urea. Random Blood Glucose. INR. -ALT, AST, Albumin and total Bilirubin. Serum Urea. Sodium level. Urine analysis. Serum urea. Alfa fetoprotein. Measurement of both Plasma and Urinary NGAL: by ELISA technique. Pelvic abdominal Ultrasound.

\section{Statistical Analysis}

SPSS software version 17 was used for statistical analysis. Qualitative (non-numerical) data were described in the form of number (frequency) and percentage whereas quantitative (numerical) data were described in the form of the mean, standard deviation (SD), and range. Analysis of variance [ANOVA] tests was used to compare quantitative dataLinear correlation coefficient was used for detection of correlation between two quantitative variables in one group. Pvalue $\leq 0.05$ was considered significant. $P$-value $<0.01$ was considered highly significant.

\section{RESULTS}

Figure (1) shows that group I included 12 males and 8 females, while in group IIA, there was 16 males and 4 females and in group IIB there was 12 males and 8 females. There was no statistical significant difference between the three groups. 


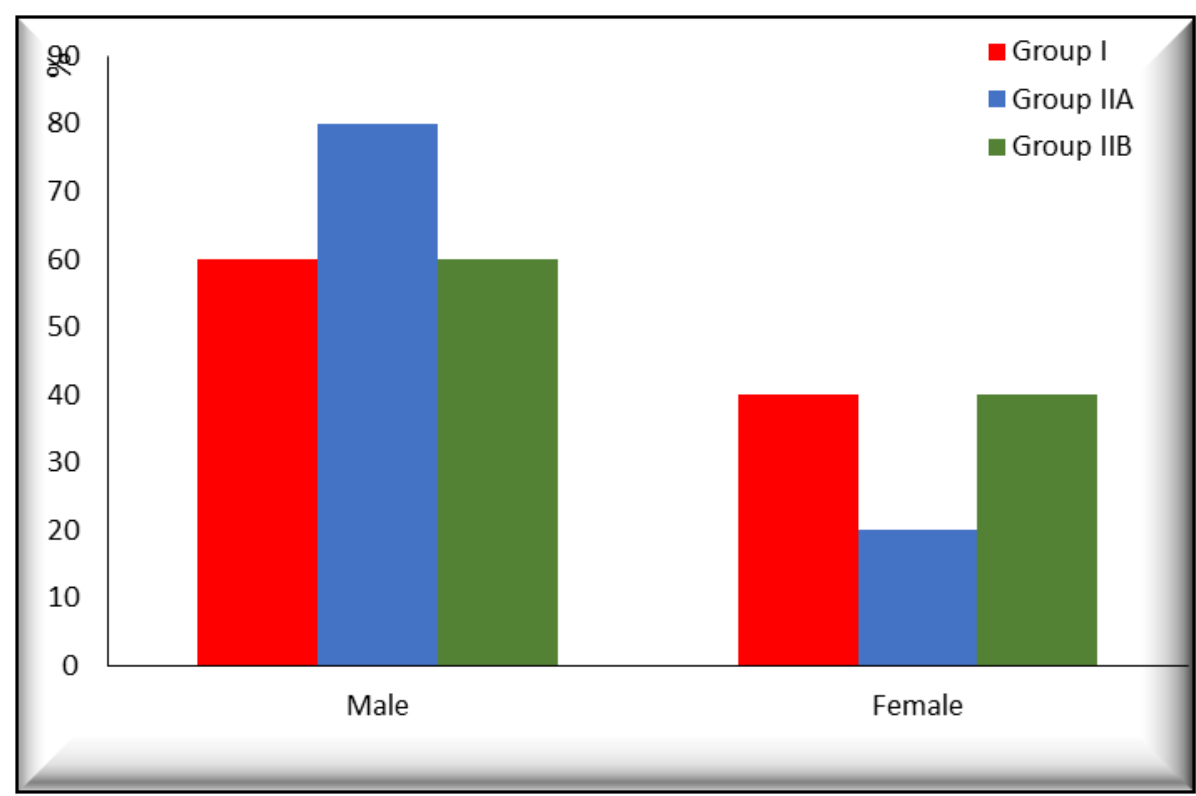

Figure (1): Comparison between different studied groups according to sex

Figure (2) showed that group I had an INR ranged from $1.1-3.2$, with a mean value of $1.66 \pm 0.5$, while group II A, ranged from 1.1 - 3 with a mean value of $1.67 \pm 0.42$ and in group II B, ranged from 1.3 -2.1 with a mean value of $1.51 \pm 0.22$. There was no significant difference between studied groups.

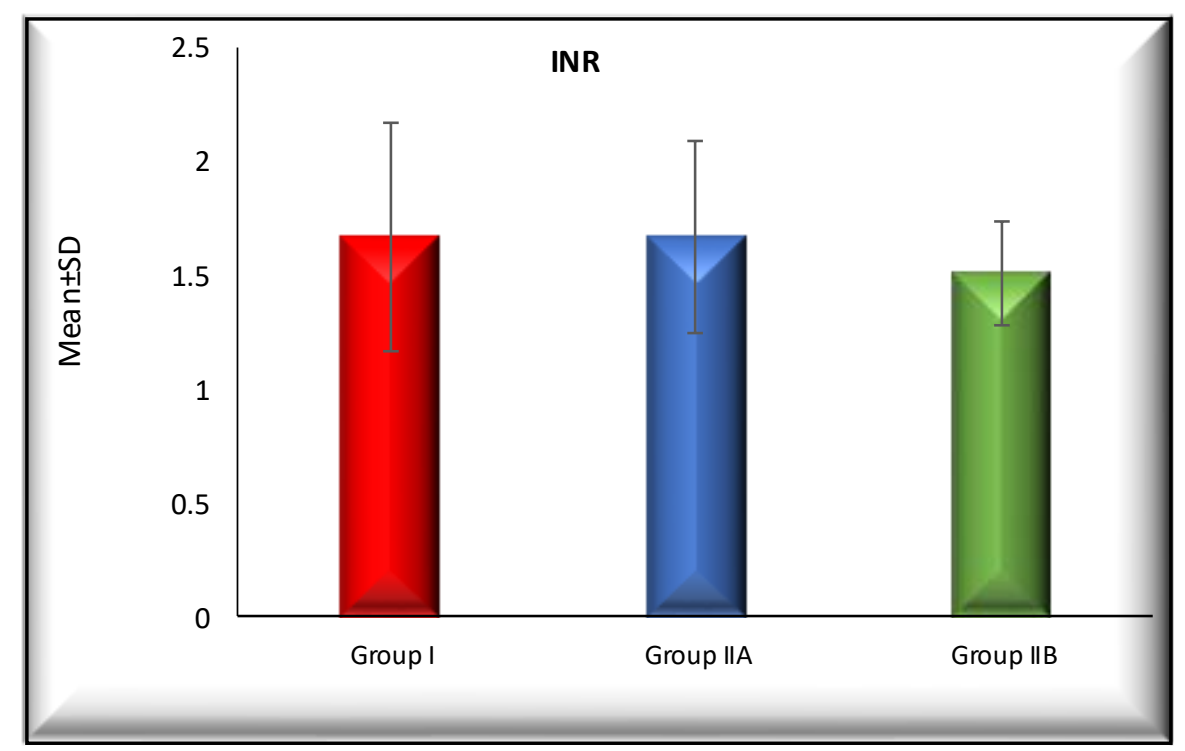

Figure (2): Comparison between different studied groups according to INR

Table (1) showed that group I had serum (S) albumin ranged from 2.1 - 3.2, while group II A, ranged from 1.9-3.4 and in group II B ranged from 1.9-3.1. There was no significant difference between groups.

Table (1): Comparison between different studied groups according to S. albumin

\begin{tabular}{|c||c|c|c|c|c||}
\hline \hline \multirow{2}{*}{$\begin{array}{c}\text { Albumin } \\
(\mathrm{g} / \mathrm{L})\end{array}$} & \multicolumn{3}{|c|}{ Groups } & \multicolumn{2}{c|}{ ANOVA } \\
\cline { 2 - 6 } & Group I (N=20) & Group IIA (N=20) & Group IIB (N=20) & F & P-value \\
\hline Mean \pm SD & $2.815 \pm 0.333$ & $2.720 \pm 0.475$ & $2.550 \pm 0.372$ & 2.276 & 0.112 \\
\hline
\end{tabular}

Table (2): showed that group I had serum bilirubin ranged from 1.2-20, while group II A, ranged from 1.9 - 20, and in group II B ranged from 2.8-9. There was no significant difference between groups.

Table (2): Comparison between different studied groups according to serum bilirubin

\begin{tabular}{|c||c|c|c||c|c|}
\hline \hline \multirow{2}{*}{$\begin{array}{c}\text { Total bilirubin } \\
(\mu \mathrm{mol} / \mathrm{L})\end{array}$} & Group I (N=20) & Group IIA (N=20) & Group IIB (N=20) & F & P-value \\
\cline { 2 - 6 } & $5.505 \pm 1.367$ & $5.705 \pm 1.351$ & $5.260 \pm 1.756$ & 0.049 & 0.952 \\
\hline \hline Mean \pm SD & &
\end{tabular}


Table (3) shows that group I had plasma NGAL ranged from 7.3-278, while group II A, ranged from 179-520 and in group II B, ranged from 288-623. There was statistical difference between group I and IIA, between group I and IIB, and between group IIA and IIB.

Table (3): Comparison between different studied groups according to Plasma Neutrophil Gelatinase Associated Lipocalin (NGAL)

\begin{tabular}{|c|c|c|c|c|c|c|c|c|}
\hline \multirow{2}{*}{$\begin{array}{c}\text { Plasma } \\
\text { NGAL } \\
\text { (g/ml) }\end{array}$} & \multicolumn{3}{|c|}{ Groups } & \multicolumn{2}{|c|}{ ANOVA } & \multicolumn{3}{|c|}{ TUKEY'S Test } \\
\hline & $\begin{array}{c}\begin{array}{c}\text { Group I } \\
(\mathrm{N}=20)\end{array} \\
\end{array}$ & $\begin{array}{c}\text { Group IIA } \\
(\mathbf{N}=\mathbf{2 0})\end{array}$ & Group IIB $(\mathbf{N}=\mathbf{2 0})$ & $\mathbf{F}$ & P-value & $\begin{array}{c}\text { I and } \\
\text { IIA }\end{array}$ & $\begin{array}{c}\text { I and } \\
\text { IIB }\end{array}$ & $\begin{array}{c}\text { IIA and } \\
\text { IIB }\end{array}$ \\
\hline $\begin{array}{c}\text { Mean } \\
\pm \text { SD }\end{array}$ & $124.315 \pm 7.793$ & $317.750 \pm 8.921$ & $392.050 \pm 17.886$ & 47.796 & $<0.001 *$ & $<0.001 *$ & $<0.001^{*}$ & $0.029 *$ \\
\hline
\end{tabular}

Table (4) shows that group I had a urinary NGAL ranged from 12 - 120, while group II A, ranged from 211493 and in group II B, ranged from 306-732. There was statistical difference between group I and IIA, between group I and IIB, and between group IIA and IIB.

Table (4): Comparison between different studied groups according to Urinary Neutrophil Gelatinase Associated Lipocalin (NGAL)

\begin{tabular}{|c|c|c|c|c|c|c|c|c|}
\hline \multirow{2}{*}{$\begin{array}{l}\text { Urinary } \\
\text { NGAL } \\
(\mathrm{mg} / \mathrm{g})\end{array}$} & \multicolumn{3}{|c|}{ Groups } & \multicolumn{2}{|c|}{ ANOVA } & \multicolumn{3}{|c|}{ TUKEY'S Test } \\
\hline & Group I & Group IIA & Group IIB & $\mathbf{F}$ & P-value & $\begin{array}{c}\text { I and } \\
\text { IIA }\end{array}$ & $\begin{array}{c}\text { I and } \\
\text { IIB }\end{array}$ & $\begin{array}{c}\text { IIA and } \\
\text { IIB }\end{array}$ \\
\hline Mean \pm SD & $61.450 \pm 6.176$ & $379.000 \pm 7.825$ & $438.050 \pm 11.269$ & 129.603 & $<0.001 *$ & $<0.001 *$ & $<0.001 *$ & 0.057 \\
\hline
\end{tabular}

Table (5) showed significant positive correlation between plasma NGAL and each of urinary NGAL, serum creatinine, urea, and INR. On the other hand, there was significant negative correlation with serum albumin.

Table (5): Correlation between Plasma NGAL and different parameters

\begin{tabular}{|c|c|c|c|c|c|c|}
\hline \multicolumn{7}{|c|}{ Correlations } \\
\hline \multirow{2}{*}{} & \multicolumn{9}{|c|}{ Plasma NGAL (g/ml) } \\
\cline { 2 - 7 } & \multicolumn{2}{|c|}{ Group I } & \multicolumn{2}{c|}{ Group IIA } & \multicolumn{2}{c|}{ Group IIB } \\
\cline { 2 - 8 } & $\mathbf{r}$ & P-value & r & P-value & r & P-value \\
\hline $\begin{array}{c}\text { Urinary NGAL } \\
(\mathrm{mg} / \mathrm{g})\end{array}$ & -0.313 & 0.179 & 0.895 & $<0.001^{*}$ & 0.846 & $<0.001^{*}$ \\
\hline Serum creatinine $(\mathrm{mg} / \mathrm{dL})$ & 0.234 & 0.321 & 0.134 & 0.572 & 0.481 & $0.032^{*}$ \\
\hline Serum urea $(\mathrm{mg} / \mathrm{dL})$ & -0.176 & 0.458 & 0.141 & 0.554 & 0.594 & $0.006^{*}$ \\
\hline INR & -0.328 & 0.158 & 0.529 & $0.017^{*}$ & 0.484 & $0.031^{*}$ \\
\hline Albumin $(\mathrm{g} / \mathrm{L})$ & 0.234 & 0.320 & -0.165 & 0.487 & -0.540 & $0.014^{*}$ \\
\hline Total bilirubin $(\mu \mathrm{mol} / \mathrm{L})$ & -0.383 & 0.096 & 0.011 & 0.963 & 0.361 & 0.118 \\
\hline
\end{tabular}

Table (6) shows that there was significant positive correlation between urinary NGAL and each of serum creatinine, urea, and serum bilirubin. On the other hand, there was significant negative correlation with serum albumin.

Table (6): Correlation between urinary NGAL and different parameters

\begin{tabular}{|c|c|c|c|c|c|c|}
\hline \multicolumn{7}{|c|}{ Correlations } \\
\hline \multirow{2}{*}{} & \multicolumn{7}{|c|}{ Urinary NGAL (mg/g) } \\
\cline { 2 - 7 } & \multicolumn{2}{|c|}{ Group I } & \multicolumn{2}{c|}{ Group IIA } & \multicolumn{2}{c|}{ Group IIB } \\
\cline { 2 - 7 } & $\mathbf{r}$ & P-value & r & P-value & r & P-value \\
\hline Serum creatinine $(\mathrm{mg} / \mathrm{dL})$ & 0.264 & 0.261 & 0.256 & 0.277 & 0.604 & $0.005^{*}$ \\
\hline Serum urea $(\mathrm{mg} / \mathrm{dL})$ & 0.127 & 0.593 & 0.130 & 0.584 & 0.690 & $0.001^{*}$ \\
\hline INR & -0.262 & 0.264 & 0.580 & $0.007^{*}$ & 0.404 & 0.077 \\
\hline Albumin $(\mathrm{g} / \mathrm{L})$ & 0.185 & 0.436 & -0.192 & 0.418 & -0.643 & $0.002^{*}$ \\
\hline Total bilirubin $(\mu \mathrm{mol} / \mathrm{L})$ & -0.006 & 0.980 & -0.029 & 0.902 & 0.491 & $0.028^{*}$ \\
\hline
\end{tabular}




\section{DISCUSSION}

Regarding gender difference, most of our candidates in both groups were males, counting for 60 $\%$ in group I (12 males) and $40 \%$ females (8). While in group IIA, males were counting for $80 \%$ (16) and $20 \%$ females (4), and in group IIB there was 12 males $(60 \%)$ and 8 females (40\%)

To evaluate hepatic condition and synthetic functions, a group of laboratory tests were done and the results are as follows, Group I had an INR a mean value of 1.66. While group II A had a mean value of 1.67 and group II B 1.51. These results show mild difference of INR between the studied groups which goes with Udgirkar et al. ${ }^{(8)}$ who enrolled 94 patients of decompensated cirrhosis and found that INR in cirrhotic patients was of mean value 1.09 while in HRS was within mean value of 1.4.

Albumin measurement showed obvious hypoalbuminemia in advanced liver disease because of failure of liver synthetic function. Group I had serum albumin with a mean value of $2.81 \pm 0.33$. While group II A had a mean value of $2.72 \pm 0.47$ and group II B 2.55 \pm 0.372 . Our study shows no significant difference between studied groups. Similarly, the study performed by Yoshikawa et al. ${ }^{(9)}$ who performed his study on 68 cirrhotic patients without renal affection with serum albumin mean value of $3.4 \pm 0.1$ and on 28 cirrhotic patients with impaired renal functions who had serum albumin mean value of $2.8 \pm 0.1$.

Total serum bilirubin measurement shows clear elevation in the studied groups, the most important reason, due to hepatocellular failure. Group I had S. bilirubin with a mean value of $5.5 \pm 5.36$ while group II A with a mean value of $5.7 \pm 5.35$ and in group II B with a mean value of $5.26 \pm 1.75$. There was an agreement with the study performed by Qasem et al. ${ }^{(10)}$, which included 160 patients with cirrhosis admitted to the Liver Units at Zagazig University Hospitals. It was found that serum bilirubin in nonascetic patient was in mean value of $2.8 \pm 1$.6, while in ascetic patients without renal impairment, S. bilirubin was in a mean value of $4.4 \pm 2.6$, and in ascetic patients with renal impairment, $S$. bilirubin was in a mean value of $6.9 \pm 4$. Similarly, Piano et al. (11) showed clear elevation of serum bilirubin in advanced liver disease due to lack of liver ability to excrete excess bile, in addition to other causes as obstruction by HCC.

Regarding Neutrophil Gelatinase-Associated Lipocalin (NGAL), our study included measurement of both plasma and urinary NGAL.

\section{Starting with Plasma NGAL:}

Group I had plasma NGAL with a mean value of $124.3 \pm 72.79$. While group II A with a mean value of $317.75 \pm 83.92$ and in group II B with a mean value of $392.05 \pm 107.88$. The elevation was relatively higher in group II B (HRS) than group II A, so, it was clear that NGAL plasma level are highly sensitive in detecting early renal dysfunction especially in combination with S. creatinine. While it's sensitivity in differentiating between the different causes of AKI is not well established because the rate of elevation is so close. This agreed with the study done by Jaques $\boldsymbol{e t} \boldsymbol{a l}$. (12), who performed his study on 105 patients and found that serum NGAL showed promising performance in early diagnosis of AKI and prognostic assessment of these patients. While in Gungor et al. (13) who aimed to investigate levels of plasma NGAL (pNGAL) and urine NGAL (uNGAL) and predictive ability of these markers for all-cause mortality, in HRS, stable cirrhosis and control subjects without renal or hepatic disease, they announced that "The results of their study showed that patients with hepatorenal syndrome had significantly higher plasma and urine NGAL levels compared with stable cirrhosis patients and control subjects. Plasma NGAL level was also significantly different between type- 1 and type- 2 HRS whereas the difference in urine NGAL did not reach statistical significance."

\section{According to urinary NGAL:}

Group I had urinary NGAL with a mean value of $61.45 \pm 36.17$. While group II A with a mean value of $379 \pm 72.825$ and in group II B with a mean value of $438.05 \pm 111.26$. There was statistical difference between studied groups. It was found that urinary NGAL is not only good predictor for early renal affection, but also it can differentiate between HRS and non HRS AKI due to the marked difference readings of its level in groups with renal impairment. According to Firu et al. ${ }^{(14)}$, there was an agreement with our study as they performed their study on 160 hospitalized patients with liver cirrhosis, and urine NGAL levels were substantially greater in patients with ascites and renal impairment compared to cirrhotic individuals who did not have renal impairment. Additionally, both urinary biomarkers were able to distinguish between the causes of AKI. A study done by Allegretti et al. ${ }^{(15)}$ showed another agreement with us, they suggested that urinary NGAL may be useful in the differential diagnosis of AKI and outcome prediction in cirrhosis. Their study approached 320 consecutive cases of AKI in cirrhosis and measured urinary NGAL. They found urinary NGAL level best distinguished ATN from other AKI, predicted AKI stage progression, and predicted 28-day mortality.

The study published by Huelin et al. ${ }^{(16)}$ goes with our results, as they performed their study on 320 cases, 153 were hypovolemia-induced AKI (48\%), 93 were HRS-AKI (29\%), 39 were ATN (12\%), and 35 were due to miscellaneous causes, and revealed that "The results show that urinary NGAL has high accuracy in the differential diagnosis between ATN and other types of AKI, including HRS-AKI and hypovolemia-induced AKI". Furthermore Lee et al. ${ }^{(17)}$ also had near results and thoughts regarding the study performed on 55 patients, with Liver cell failure who exhibited acute kidney injury upon admission. Their 
result showed that urinary NGAL has emerged as a potential diagnostic biomarker for AKI. Its level was significantly higher in ATN patients than in non-ATN patients and was useful for detecting AKI early in hospitalized cirrhotic patient and differentiation of ATN from HRS. In his study, the urinary NGAL range differed greatly according to the AKI etiology

Another agreement with Udgirkar et al. (8) who performed his study on 94 patients of decompensated cirrhosis. uNGAL was tested in all patients upon admission to the hospital, and it was discovered that uNGAL at baseline can help distinguish between HRS, prerenal AKI, and iAKI in cirrhotic patients when sCr levels are ineffective. Patients with higher uNGAL levels had higher transplant-free mortality at 30 days.

On the other hand, in contrast to our study, Alhaddad et al. ${ }^{(18)}$ who performed their study on 90 patients with cirrhosis, announced that "In our study only the pNGAL level was statistically different between the groups contrary to uNGAL. As to why the uNGAL did not increase, we have no explanation".

In conclusion, both plasma and urinary NGAL are good predictors in early renal impairment in cirrhotic patients.

Furthermore, urinary NGAL can differentiate between different types of acute kidney injury, while serum NGAL level in different types of AKI is so close, so it can't differentiate between them.

\section{CONCLUSION}

Both plasma and urinary NGAL levels are markedly elevated in renal affection whatever the cause, so they are highly sensitive in detecting early renal impairment. However, the rate of elevation of plasma NGAL is so close in different types of AKI so it's not advisable to use it alone to diagnose HRS. On the other hand, Urinary NGAL shows noticeable differences between degree of elevation with different causes of AKI, so it's somehow could be a trusted diagnostic tool to diagnose HRS.

\section{Financial support and sponsorship: Nil. \\ Conflict of interest: Nil.}

\section{REFERENCES}

1. Kaushal G, Shah S (2014): Challenges and advances in the treatment of AKI. Journal of the American Society of Nephrology, 5:877-883.

2. Hartleb M, Gutkowski K (2012): Kidneys in chronic liver diseases. World Journal of Gastroenterology: WJG., 24:3035-3039.

3. Choi Y, Kim J, Koo J et al. (2014): Prevalence of renal dysfunction in patients with cirrhosis according to ADQI-IAC working party proposal. Clinical and molecular Hepatology, 20(2):185-191.
4. Wong F, Nadim M, Kellum J et al. (2011): Working Party proposal for a revised classification system of renal dysfunction in patients with cirrhosis. Gut, 60(5):702709.

5. Lopes J, Jorge $S$ (2013): The RIFLE and AKIN classifications for acute kidney injury: a critical and comprehensive review. Clinical Kidney Journal, 6.1:814.

6. Arroyo V (2013): Acute kidney injury (AKI) in cirrhosis: should we change current definition and diagnostic criteria of renal failure in cirrhosis. J Hepatol., 59:415-417.

7. Fagundes C, Barreto R, Guevara $M$ et al. (2013): A modified acute kidney injury classification for diagnosis and risk stratification of impairment of kidney function in cirrhosis. Journal of Hepatology, 59.3:474-481.

8. Udgirkar S, Rathi P, Sonthalia N et al. (2020): Urinary neutrophil gelatinase-associated lipocalin determines short-term mortality and type of acute kidney injury in cirrhosis. JGH Open, 4(5):970-7.

9. Yoshikawa K, Iwasa M, Eguchi A et al. (2017): Neutrophil gelatinase-associated lipocalin level is a prognostic factor for survival in rat and human chronic liver diseases. Hepatology Communications, 1(9):94656.

10. Qasem A, Farag S, Hamed E et al. (2014): Urinary biomarkers of acute kidney injury in patients with liver cirrhosis. https://www.hindawi.com/journals/isrn/2014/376795/

11. Piano S, Rosi S, Maresio G et al. (2013): Evaluation of the Acute Kidney Injury Network criteria in hospitalized patients with cirrhosis and ascites. Journal of Hepatology, 59(3):482-9.

12. Jaques D, Spahr L, Berra G et al. (2019): Biomarkers for acute kidney injury in decompensated cirrhosis: A prospective study. Nephrology, 24(2):170-80.

13. Gungor G, Ataseven H, Demir A et al. (2014): Neutrophil gelatinase-associated lipocalin in prediction of mortality in patients with hepatorenal syndrome: a prospective observational study. Liver International, 34(1):49-57.

14. Firu S, Streba C, Firu D et al. (2013): Neutrophil Gelatinase Associated Lipocalin (NGAL)-a biomarker of renal dysfunction in patients with liver cirrhosis: Do we have enough proof?. Journal of Medicine and Life, 8:15-20.

15. Allegretti A, Solà E, Ginès $P$ (2020): Clinical application of kidney biomarkers in cirrhosis. American Journal of Kidney Diseases, 76: 710-719.

16. Huelin P, Solà E, Elia $C$ et al. (2019): Neutrophil gelatinase-associated lipocalin for assessment of acute kidney injury in cirrhosis: a prospective study. Hepatology, 70(1):319-33.

17. Lee J, Yoon E, Park S et al. (2019): Clinical significance of urinary neutrophil gelatinase-associated lipocalin levels in defining the various etiologies of acute kidney injury in liver cirrhosis patients. The Korean Journal of Gastroenterology, 74(4):212-8.

18. Alhaddad O, Alsebaey A, Amer M et al. (2015): Neutrophil gelatinase-associated lipocalin: a new marker of renal function in C-related end stage liver disease. https://pubmed.ncbi. nlm.nih.gov/26221137/ 